\section{Resultados chilenos del registro internacional de factores de riesgo $y$ tratamiento de angina inestable e infarto al miocardio sin supradesnivel del segmento ST: ACCORD (ACute CORonary syndrome Descriptive study)}

\author{
BENJAMÍN STOCKINS ${ }^{1}$, FRANCISCO ALBORNOZ², DARÍO MARTÍNEZ ${ }^{3}$, \\ PABLA CAMPOS ${ }^{4}$, JORGE GAJARDO 5 , RUBÉN LAMICH ${ }^{6}$, \\ LEOPOLDO MANRÍQUEZ ${ }^{7}$, VÍCTOR PÉREZ ${ }^{8}$, PAMELA ROJO9, \\ PABLO SEPÚLVEDA ${ }^{10}$, M. GABRIELA PUMARINO ${ }^{11}$, RAMÓN CORBALÁN ${ }^{12}$
}

\section{Chilean results of the international registry of} risk factors and treatment of unstable angina and non ST elevation myocardial infarction: ACCORD (ACute CORonary syndrome Descriptive study)

Background: Guidelines for the management of unstable angina (UA) and non ST elevation myocardial infarction (NSTEMI) have been issued, however current practices are unknown in Chile. Aim: To evaluate in a prospective cohort of NSTEMI patients the current practices, treatments and risk factors. Material and Methods: One year prospective International non interventional registry, conducted in Chile between January 2005 and November 2006. Results: Two hundred thirty three Chilean NSTEMI patients were enrolled. Mortality was $5.5 \%$ at the end of the follow-up. Mean age was 61.6 years, and 30.6\% were female. Most of the patients had at least one risk factor (98\%): hypertension (84\%), previous myocardial infarction (33\%), dyslipidemia (54\%), diabetes (33\%), current smoking (30\%). Main procedures during the hospitalization were coronary angiogram (67\%), angioplasty (33\%; 88\% with stent) and coronary bypass surgery (7\%). During procedures, $31 \%$ of patients received clopidogrel, and $4.2 \%$ glycoprotein IIb/IIIa antagonists. Medical management was selected for $60 \%$ of patients. In comparison to men, women received less interventional procedures despite having more risk factors. Treatments prescribed at discharge were aspirin (97\%), clopidogrel (49\%), beta blockers (78\%), diuretics (21\%), lipid lowering agents (78\%), oral hypoglycemic agents (13\%) and insulin (9\%). At the end of the 1-year follow-up, treatments were aspirin (84\%), beta blockers (72\%), diuretics (19\%), and dual antiplatelet therapy with clopidogrel (16\%). Conclusions: A high prevalence of multiple risk factors for cardiovascular disease in Chilean patients with NSTEMI was observed. More aggressive primary and secondary preventive measures are urgently needed. Use of therapies proposed in the guidelines is high, but dual antiplatelet therapy is less than 50\% at discharge and decreases during the one year-follow-up.

(Rev Med Chile 2011; 139: 19-26).

Key words: Coronary artery disease; Myocardial infarction; Risk factors.

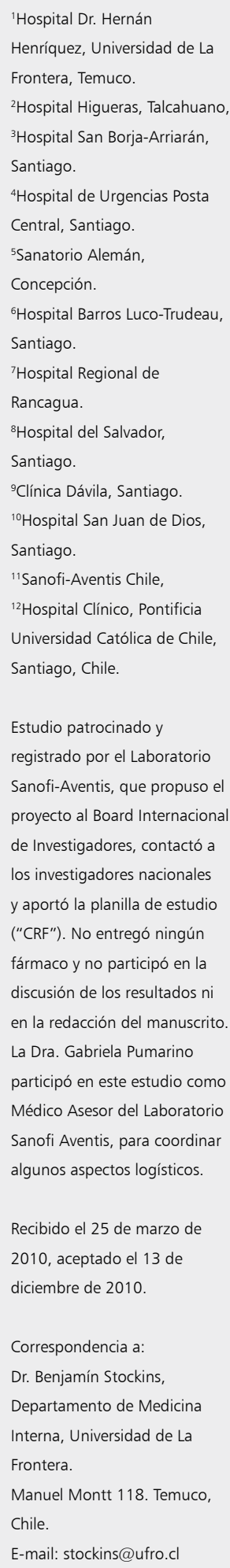


I a enfermedad coronaria es una importante y creciente causa de morbilidad y mortalidad en el mundo, así como también en Chile ${ }^{1,2}$. La lesión coronaria es un proceso dinámico, que se inicia con el daño endotelial y la formación de la placa ateroesclerótica. La placa puede permanecer estable o ser afectada por un proceso variable de trombosis, que limita o detiene el flujo coronario ${ }^{3}$. Los cambios de flujo llevan a manifestaciones clínicas de diversa magnitud, que requieren una aproximación terapéutica agresiva, controlada y basada en la evidencia disponible. La angina inestable (AI) y el infarto agudo al miocardio sin supradesnivel del segmento ST (IAMSST) se reconocen como manifestaciones clínicas frecuentes e importantes de la enfermedad coronaria bajo la forma de un Síndrome Coronario Agudo sin elevación del segmento ST (SCASST). Ellas presentan un pronóstico más ominoso en comparación con la angina crónica estable, por lo que existen normas para su manejo ${ }^{4}$. El mayor riesgo de un evento cardiovascular grave posterior, incluyendo la muerte, ocurre precozmente y se mantiene a largo plazo en diferentes magnitudes, dependiendo del diagnóstico, factores de riesgo, tratamientos y plazos, entre otros ${ }^{5,6}$.

Se han editado guías de tratamiento para estas condiciones, que establecen esquemas terapéuticos, con sus respectivos grados de recomendación ${ }^{7-9}$. Los puntos centrales en estas guías son el adecuado manejo de la terapia anti trombótica, el tratamiento anti isquémico, el uso de anticoagulantes y el restablecimiento del flujo coronario ${ }^{7-9}$. Existe escasa información respecto al grado de adherencia a tales tratamientos en la práctica clínica.

El objetivo de este estudio fue evaluar en una cohorte prospectiva de pacientes con SCASST el uso en la práctica clínica de terapia de probada eficacia (en especial de terapia anti trombótica), además de obtener información de las características demográficas y factores de riesgo de estos pacientes en nuestro país.

\section{Material y Método}

Diseño: Este estudio corresponde a los datos chilenos de un registro internacional no publicado. Fue un registro no intervencional, realizado en 16 países entre los años 2004 y 2006. En Chile, participaron 12 centros hospitalarios (10 hospitales públicos, 1 privado y 1 universitario). El registro se realizó desde enero de 2005 a noviembre de 2006.

Comprendió un total de 5 visitas, siendo la primera previa alta del evento agudo, y luego un seguimiento a los 3, 6, 9 y 12 meses. Se efectuó un registro protocolizado, estandarizado y con monitoreo de todos los datos obtenidos.

Los criterios de inclusión fueron: Pacientes mayores de 21 años hospitalizados por dolor torácico debido a un SCASST. El diagnóstico de AI y de IAMSST fue realizado sobre la base de definiciones aceptadas internacionalmente y de otros registros nacionales, vale decir la presencia de un cuadro clínico de dolor anginoso de reposo o prolongado, 48 horas antes del ingreso, o cambios del segmento ST o la onda T o elevación de marcadores de daño miocárdico ${ }^{7}$.

Se excluyeron pacientes que al ingreso se presentaron con infarto agudo al miocardio con supradesnivel del segmento $\mathrm{ST}$.

Los pacientes fueron tratados de acuerdo a las prácticas clínicas locales establecidas en cada centro y/o a las de sus médicos tratantes. La hospitalización se realizó en Unidades Coronarias, Unidades de Cuidados Intensivas generales o salas de Medicina Interna, de acuerdo a condiciones locales.

Las instituciones participantes contaban con distinto grado de complejidad y accesibilidad a procedimientos angiográficos invasivos durante el año que duró el registro. La decisión sobre la realización y precocidad de un estudio angiográfico se hizo sobre la base de los protocolos de cada centro.

Se registraron: Datos demográficos; Historia de eventos vasculares cardíacos y cerebrales; Factores de riesgo; Síntomas y características del episodio agudo; Electrocardiograma; Función ventricular izquierda, evaluada por Fracción de Eyección, la que fue medida por ecocardiografía, medicina nuclear o angiografía; Exámenes de laboratorio relacionados al evento; Factores de riesgo; Procedimientos y medicamentos cardiovasculares durante y previos al evento agudo; y el Tratamiento médico y sobrevida de los pacientes durante el seguimiento.

El protocolo de estudio fue sometido a revisión por un Comité de Ética internacional y el Comité Ético Científico del Servicio de Salud Araucanía Sur (Temuco). Se obtuvo el consentimiento informado de todos los pacientes. 
Factores de riesgo y tratamiento en cardiopatía coronaria - B. Stockins et al

\section{Resultados}

\section{Características demográficas de los pacientes}

El registro internacional enroló 6.868 pacientes en 16 países. En Chile ingresaron 233 pacientes, en 12 centros. El 85,4\% de los pacientes completó la

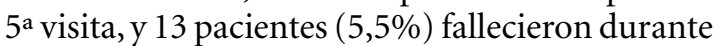
el seguimiento a 1 año.

Características clínicas de los pacientes (Tabla 1): El promedio de edad fue de 61,6 ( \pm DE 11,9) años y $30,6 \%$ fueron mujeres.

$\mathrm{Al}$ ingreso, $84,1 \%$ presentó dolor torácico típico. La frecuencia cardiaca promedio de ingreso fue de $76( \pm 18)$ latidos por minuto, y la presión arterial promedio de $146( \pm 28) / 84( \pm 17) \mathrm{mm} \mathrm{Hg}$. El 95\% de los pacientes presentaba ritmo sinusal.

El diagnóstico al alta fue de AI en 67\%, IAMSST en 31,5\% e infarto con supradesnivel del segmento ST en 1,3\% de los pacientes.

Al 60\% de los pacientes se les midió la fracción de eyección durante el episodio índice, siendo en el $80 \%$ de ellos normal (mayor a 50\%). El 15\% mostraba un deterioro leve de ella (entre 40 y 50\%).

\section{Factores de riesgo}

El 98,3\% de los pacientes tenía factores de riesgo. De ellos, $85,4 \%$ tenía al menos uno, destacando: hipertensión arterial en $84 \%, 33 \%$ con antecedente de un infarto agudo del miocardio previo y $22 \%$ tenía historia familiar de eventos cardiovasculares. El 5,2\% tenía enfermedad cerebrovascular (accidente cerebrovascular isquémico, endarectomía carotídea o crisis isquémica transitoria) (Tabla 1 ).

Entre los pacientes que tenían al menos un factor de riesgo destacan: dislipidemia en 54\%, diabetes mellitus en 33\%, tabaquismo actual en $30 \%$, y tabaquismo previo en $38 \%$. Un porcentaje importante de pacientes tenía sobrepeso $\mathrm{u}$ obesidad: IMC entre $25-30 \mathrm{~kg} / \mathrm{m}^{2}: 45 \%$ e IMC $^{3}$ $30 \mathrm{~kg} / \mathrm{m}^{2}: 27 \%$.

El 58\% de los pacientes estaba recibiendo ácido acetilsalicílico previo al ingreso.

El 97\% de los pacientes presentaba un factor de riesgo, y aproximadamente la mitad de ellos (47\%), presentaba 4 o más factores de riesgo simultáneamente (Figura 1).

\section{Procedimientos durante la hospitalización}

A $67,3 \%$ de los pacientes se le realizó una coronariografía, durante el evento agudo. El $33 \%$ de los pacientes recibió una angioplastía, implantándose al menos un stent en $88 \%$ de ellas. Este procedimiento fue realizado en promedio 4,8 días después del ingreso. En 7\% se realizó cirugía de revascularización coronaria, la que se efectuó en promedio a los 8 días con 2,8 puentes aortocoronarios por paciente. El $60 \%$ de los pacientes recibió sólo tratamiento médico no intervencional.

Durante el cateterismo, $31,3 \%$ recibió clopidogrel (de ellos, $23 \%$ lo recibió antes, $36 \%$ después y $40 \%$ antes y después del procedimiento). De

Tabla 1. Características clínicas de los pacientes incluidos en el estudio ACCORD

\begin{tabular}{|c|c|}
\hline Número de pacientes & 233 \\
\hline Edad (años; promedio $\pm \mathrm{DE}$ ) & $61,6 \pm 11,9$ \\
\hline Mujeres (\%) & 30,6 \\
\hline IMC $25-30$ kg/m² (\%) & 45,5 \\
\hline $\mathrm{IMC}>30 \mathrm{~kg} / \mathrm{m}^{2}(\%)$ & 27 \\
\hline \multicolumn{2}{|l|}{ Historia y factores de riesgo (\%) } \\
\hline Historia de enfermedad cardiovascular & 85 \\
\hline Hipertensión arterial & 84 \\
\hline Infarto agudo al miocardio previo & 33 \\
\hline Angina inestable & 17 \\
\hline Angina estable & 12 \\
\hline $\mathrm{PCl}$ & 13 \\
\hline Insuficiencia cardiaca crónica & 8,5 \\
\hline Disrritmia & 4,0 \\
\hline Historia familiar de eventos CV & 22 \\
\hline $\begin{array}{l}\text { Historia de enfermedad } \\
\text { cerebrovascular }(\%)\end{array}$ & 5,2 \\
\hline Otros factores de riesgo (\%): & 88 \\
\hline Dislipidemia & 54 \\
\hline Diabetes mellitus & 33 \\
\hline Tabaquismo activo & 30 \\
\hline Tabaquismo previo & 38 \\
\hline Acido acetilsalicílico previo & 58 \\
\hline Dolor torácico típico & 84,1 \\
\hline \multicolumn{2}{|l|}{ Diagnóstico al alta (\%) } \\
\hline Angina Inestable & 67 \\
\hline IAMSST & 32 \\
\hline IAMCST & 1 \\
\hline
\end{tabular}

IMC: Índice de masa corporal; PCI: Intervención percutánea coronaria; CV: cardiovascular; DE: desviación estándar. 


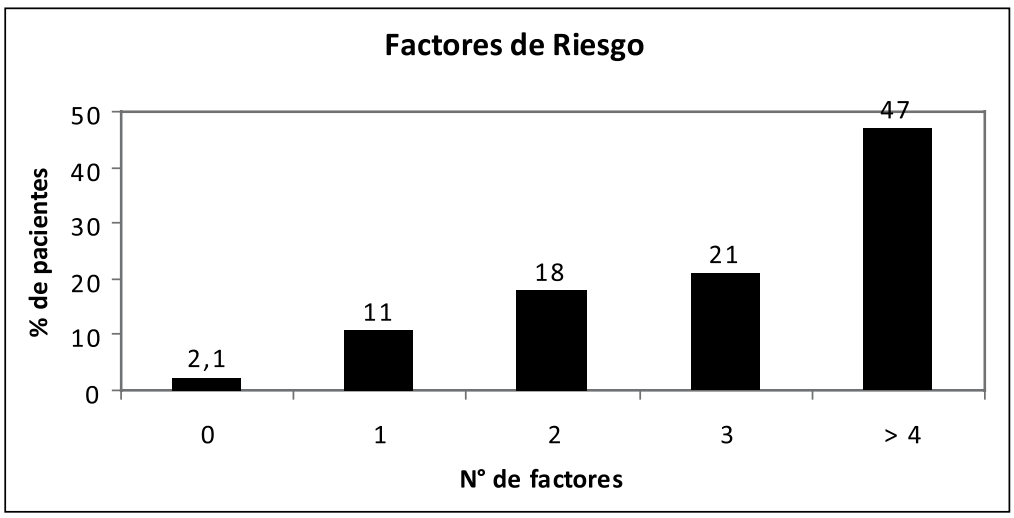

Figura 1. Número de factores de riesgo por pacientes en el estudio ACCORD.

Tabla 2. Modalidad de tratamiento aplicado durante la hospitalización en los pacientes del estudio ACCORD

\begin{tabular}{|lccc|}
\hline & Médico & Angioplastía & Cirugía \\
\hline Número (\%) & $140(60)$ & $76(33)$ & $17(7)$ \\
\hline Edad (años; promedio \pm DE) & $63 \pm 11,8$ & $58 \pm 11,7$ & $66 \pm 8,4$ \\
Mujeres (\%) & 38 & 23 & 6 \\
Antec enf coronaria (\%) & 47 & 87 & 47 \\
ASA previo ingreso (\%) & 69 & 54 & 65 \\
Hipertensión arterial (\%) & 77 & 61 & 82 \\
Dislipidemia (\%) & 41 & 57 & 64 \\
Diabetes (\%) & 32 & 17 & 41 \\
\hline
\end{tabular}

aquellos que lo recibieron antes del cateterismo, $21,7 \%$ no recibió una dosis de carga. El 4,3\% de los pacientes recibió un antagonista de GPIIb/IIIa.

La Tabla 2 muestra las características de los grupos de acuerdo a la estrategia terapéutica escogida (tratamiento médico o revascularización). Se observa una mayor tendencia al predominio de varones en los pacientes sometidos a revascularización y menor edad en los sometidos a angioplastía. Al grupo de mujeres se les realizaron menos procedimientos, pese a que cuando se hace el análisis por sexo, ellas presentan más factores de riesgo como hipertensión y diabetes.

Tratamiento durante la hospitalizacion y alta

La Tabla 3 muestra el uso de fármacos al alta y a los 12 meses de seguimiento. La aspirina fue utilizada en el 97\% (dosis promedio: $157 \mathrm{mg} /$
Tabla 3. Porcentaje de uso de fármacos al alta y al año de seguimiento en pacientes del estudio ACCORD

\begin{tabular}{|lcc|}
\hline & $\begin{array}{c}\text { Al alta } \\
\mathbf{\%}\end{array}$ & $\begin{array}{c}\text { Al año } \\
\mathbf{\%}\end{array}$ \\
\hline Aspirina & 97 & 84 \\
Clopidogrel & 49 & 16 \\
Beta bloqueantes & 78 & 72 \\
\hline Heparina de BPM & 21 & --- \\
\hline Heparina no fraccionada & 66 & --- \\
\hline Hipolipemiantes & 78 & 79 \\
\hline Diuréticos & 21 & 19 \\
\hline Hipoglicemiantes orales & 13 & 19 \\
\hline Insulina & 9,2 & 8,7 \\
\hline
\end{tabular}


Tabla 4. Medicamentos al alta según la modalidad de tratamiento recibido por los pacientes del estudio ACCORD

\begin{tabular}{|lccc|}
\hline & $\begin{array}{c}\text { Médico } \\
\text { \% }\end{array}$ & $\begin{array}{c}\text { Angioplastía } \\
\text { \% }\end{array}$ & $\begin{array}{c}\text { Cirugía } \\
\text { \% }\end{array}$ \\
\hline Aspirina & 96 & 97 & 94 \\
Estatinas & 71 & 85 & 88 \\
Heparina no fraccionada & 73 & 49 & 47 \\
Heparina de bajo peso molecular & 12 & 35 & 35 \\
Beta Bloqueadores & 80 & 85 & 100 \\
Clopidogrel & 30 & 95 & 0 \\
Nitratos & 67 & 54 & 82 \\
\hline
\end{tabular}

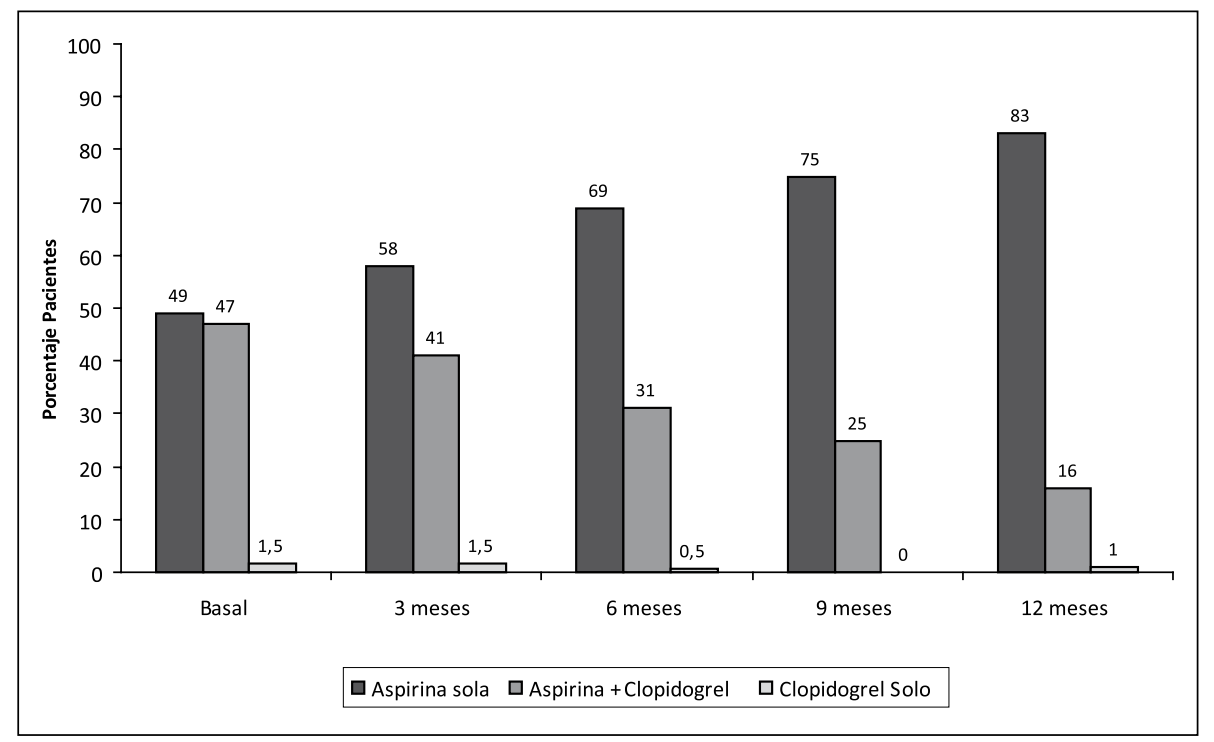

Figura 2. Uso de terapia antiplaquetaria al alta en pacientes con SCASST.

día), clopidogrel en $49 \%$, betabloqueadores en $78 \%$, diuréticos en $21 \%$, hipolipemiantes en $78 \%$, hipoglicemiantes orales en $13 \%$ e insulina en $9 \%$.

La mayor parte de los pacientes recibió algún tipo de heparina (no fraccionada o de bajo peso molecular), predominando el uso de la forma no fraccionada (76\% de los pacientes), en especial en los pacientes con la opción de tratamiento médico. Heparina de bajo peso molecular sólo la recibió el $21 \%$ del total de pacientes. No existió una diferencia significativa en la mayor parte de los otros medicamentos recibidos, salvo en el uso de clopidogrel, el cual se concentró en los pacientes sometidos a angioplastía, que lo recibieron en el 95\% de los casos, comparado con sólo 30\% de aquellos con la estrategia de tratamiento médico (Tabla 4).

\section{Tratamiento durante la evolución}

Medicamentos al año de seguimiento (Tabla 3): aspirina $84 \%$, betabloqueadores $72 \%$, diuréticos $19 \%$, hipolipemiantes $79 \%$, hipoglicemiantes orales $19 \%$, e insulina $8,7 \%$. Clopidogrel fue utilizado en $39 \%$ de los pacientes a los tres meses y en $14 \%$ al año de seguimiento.

La Figura 2 muestra el uso simultáneo de aspirina y clopidogrel en el tiempo, con una disminución progresiva de la terapia dual, llegando a sólo $16 \%$ al completar el año de seguimiento. 


\section{Discusión}

El registro ACCORD-CHILE muestra algunos hechos fundamentales:

- Existe una alta prevalencia de factores de riesgo coronario en pacientes que ingresan con diagnóstico de SCASST.

- Las mujeres reciben menos procedimientos de revascularización, pese a tener en mayor proporción algunos factores de riesgo.

- Existe una alta utilización global de fármacos probadamente útiles.

- La utilización de aspirina es frecuente, pero hay un uso escaso de otros antiplaquetarios, lo que no es concordante con las normas de tratamiento.

- Un alto número de pacientes sólo recibe tratamiento médico (sin un procedimiento de revascularización), siendo ellos los que reciben menos tratamiento con fármacos.

\section{Demografía y factores de riesgo}

La proporción entre pacientes con AI/IAMSST fue similar a la de otras series ${ }^{10}$. Las características poblacionales de este grupo fueron similares al registro nacional previo, realizado entre los años 2000 y 2002 por Corbalán et al ${ }^{11}$, pero la edad de aparición del evento en este registro fue cuatro años menor. Asimismo existe una mayor prevalencia de obesidad, diabetes mellitus, hipertensión arterial, mayor proporción de infartos antiguos, accidentes vasculares encefálicos, eventos en pacientes recibiendo aspirina, que junto con una mayor letalidad sugieren que esta serie podría comprender pacientes de mayor gravedad, siendo similar a registros internacionales como el Euro Heart Survey ${ }^{12}$. Es posible que en la actualidad se esté recibiendo un mayor número de pacientes tratados previamente por un evento coronario agudo, que quedan con mayor riesgo de eventos posteriores.

\section{Tratamiento farmacológico}

Este registro muestra el uso de fármacos de probada eficacia durante la hospitalización. Los pacientes recibieron en un alto porcentaje aspirina, estatinas, heparina y bloqueadores beta adrenérgicos. Hay un aumento del uso de estatinas durante el episodio agudo, probablemente por la incorporación de la evidencia aportada por estudios que muestran los efectos precoces de las estatinas ${ }^{13}$.
Hace excepción al seguimiento de las guías, el mayor uso de heparina no fraccionada, en comparación con las de bajo peso molecular recomendadas en ellas y el bajo uso de clopidogrel, lo que puede explicarse en parte por un problema de costo, que hace a estos dos fármacos menos accesibles al uso rutinario en los hospitales públicos.

La adherencia al año de tratamiento es extremadamente alta para algunos medicamentos: más de $70 \%$ para beta bloqueadores y estatinas, y de $84,5 \%$ para aspirina. Estas cifras sugieren la existencia de programas específicos de prevención secundaria que explicarían este alto nivel de cumplimiento.

El 20\% de los diabéticos conocidos no recibe farmacoterapia al alta. Hay escasa variación en las otras terapias. No se evaluó el uso de inhibidores de la enzima convertidora ni de inhibidores del receptor de la angiotensina al año por un problema en el diseño de instrumento de registro.

\section{Uso de antiplaquetarios}

En el manejo agudo del SCASST, el uso de aspirina está ampliamente difundido, lo que se mantiene en forma importante al año de seguimiento (al año, el uso de aspirina disminuye sólo en $12 \%$ ). El clopidogrel, en cambio, es usado en aproximadamente la mitad de los pacientes en la etapa aguda, concentrándose su uso en aquellos que son sometido a angioplastia y a los cuales se les implanta un stent. Aun así, sólo el 23\% lo recibe antes del cateterismo y sólo un tercio de los pacientes recibió clopidogrel o antagonista GPIIb/IIIa durante el procedimiento. Al año, el uso de clopidogrel disminuye en $71 \%$ (Figura 2). Los pacientes que reciben tratamiento médico (no revascularización) son los que menos reciben clopidogrel y, cuando lo utilizan, es por períodos más breves. Ello se contradice con las guías basadas en dos grandes estudios. El estudio CURE ${ }^{5}$ demostró el beneficio de agregar clopidogrel a la terapia estándar en pacientes con SCASST, y sobre la base de estos hallazgos, en 2002, la Asociación Americana de Cardiología (AHA) y el Colegio Americano de Cardiología (ACC) establecen en sus guías la recomendación del inicio inmediato de clopidogrel en pacientes con AI y IAMSST y el prolongarlo por un año ${ }^{14}$. Adicionalmente el estudio CRUSADE ${ }^{15}$, que evaluó el uso precoz de clopidogrel en IAMSST, mostró que sólo el $38 \%$ de los pacientes que no va a intervención percutánea recibe clopidogrel pre- 
cozmente. Sus resultados demostraron que el uso precoz del clopidogrel se asoció a menores tasas de complicaciones intrahospitalarias, tales como muerte, infarto del miocardio, shock cardiogénico, insuficiencia cardiaca o accidente vascular encefálico, comparado con el uso tardío del fármaco (después de 24 horas).

La falta de traspaso de las guías a la práctica clínica, no es exclusivo de nuestro país, ya que numerosos registros muestran hechos similares $^{16,17}$. En nuestro país ha jugado un rol, en este uso insuficiente, el que algunos servicios públicos no contaban en dicha fecha con el medicamento, pese a las evaluaciones costo-efectividad favorables para el uso de la terapia antiplaquetaria dual ${ }^{18}$ independientemente del tipo de terapia utilizada (intervencionista o médica).

\section{Tratamiento de revascularización}

El porcentaje de pacientes sometidos a un estudio coronario fue de $67,4 \%$. El $40 \%$ del total de la serie recibió tratamiento de revascularización, siendo éste mayoritariamente endovascular (33\%).

Esta cifra es algo mayor a la reportada por Corbalán et al el año 2004 (52\% de revascularización), donde se observó un mayor porcentaje de pacientes sometidos a cirugía ${ }^{11}$.

Los pacientes con tratamiento médico, tendieron a tener una edad más avanzada que aquellos a los que se les realizó angioplastía (63 vs 58 años). Igualmente existió una mayor proporción de mujeres en los pacientes tratados médicamente ( $38 v \mathrm{~s}$ $23 \%$ ). La menor proporción de mujeres con tratamiento invasivo ha sido observada en numerosas series tanto en Chile como en el extranjero. En Chile, el registro GEMI mostró que las pacientes de sexo femenino con infarto de miocardio con supradesnivel de segmento ST reciben menos procedimientos probadamente efectivos en comparación con los varones ${ }^{19}$.

En relación al estudio angiográfico precoz de los pacientes, se pudo apreciar que 67,3\% de los pacientes fue estudiado durante la hospitalización y 33\% recibió revascularización por angioplastia. Estas cifras son concordantes con series extranjeras. El grupo de pacientes estadounidenses del registro CRUSADE fue estudiado en 63,5\% y recibió angioplastía el $37,8 \%{ }^{20}$.

El aumento del acceso a procedimientos invasivos, por el mayor número de laboratorios de cateterismo cardíaco, así como un financiamiento por parte del sistema público chileno, probablemente contribuirán a que las cifras de angioplastías sean progresivamente superiores tanto en la actualidad como en el futuro.

\section{Conclusiones}

El registro muestra un manejo general adecuado de los pacientes con SCASST en los hospitales en los cuales se efectuó el registro. Los pacientes reciben fármacos de probada eficacia, tanto durante la hospitalización como al año de seguimiento. Sin embargo, un punto débil de la terapia lo constituye la falta de inhibición plaquetaria dual (aspirina-clopidogrel), incorporada en las guías de tratamiento, que ha demostrado una alta efectividad tanto en pacientes sometidos a tratamiento médico como a revascularización miocárdica.

La existencia de una creciente cantidad de factores de riesgo en pacientes con AI y con IAMSST requiere hacer aún más énfasis en prevención primaria y secundaria de la enfermedad coronaria, probablemente con programas específicos que se apliquen en forma sistemática y rigurosa.

Este registro tiene limitaciones, como es el bajo número de pacientes, que no permite obtener conclusiones más categóricas dentro de los subgrupos donde se muestran algunas tendencias.

\section{Referencias}

1. Murray CJ, López AD. Alternative proyections of mortality and disability by cause 1990-2020: Global Burden of Disease Study. Lancet 1997; 349: 1498-504.

2. Solimano CG, Mazzei PM. [Which are the causes of death among Chileans today? Long term perspectivas]. Rev Med Chile 2007; 135: 932-8.

3. Dangas G, Mehran R, Wallenstein S, Courcoutsakis NA, Kakarala V, Hollywood J, et al. Correlation of angiographic morphology and clinical presentation in unstable angina. J Am Coll Cardiol 1997; 29: 519-25.

4. Lagerqvist B, Husted S, Kontny F, Ståhle E, Swahn E, Wallentin L; Fast Revascularisation during InStability in Coronary artery disease (FRISC-II) Investigators. 5 -year outcomes in the FRISC-II randomised trial of an invasive versus a non-invasive strategy in non-STelevation acute coronary syndrome: a follow-up study. Lancet 2006; 368: 998-1004.

5. Yusuf S, Zhao F, Mehta SR, Chrolavicius S, Tognoni G, Fox KK. Effects of clopidogrel in addition to aspirin in patients with acute coronary syndromes without STsegment elevation. N Engl J Med 2001; 345: 494-502. 
6. Steinhubl SR, Berger PB, Mann JT 3rd, Fry ET, DeLago A, Wilmer C, et al. Early and sustained dual oral antiplatelet therapy following percutaneous coronary intervention: a randomized controlled trial. JAMA 2002; 288: 2411-20.

7. Anderson JL, Adams CD, Antman EM, Bridges CR, Califf RM, Casey DE Jr, et al. ACC/AHA 2007 guidelines for the management of patients with unstable angina/non ST-elevation myocardial infarction: a report of the American College of Cardiology/American Heart Association Task Force on Practice Guidelines (Writing Committee to Revise the 2002 Guidelines for the Management of Patients With Unstable Angina/Non ST-Elevation Myocardial Infarction): developed in collaboration with the American College of Emergency Physicians, the Society for Cardiovascular Angiography and Interventions, and the Society of Thoracic Surgeons: endorsed by the American Association of Cardiovascular and Pulmonary Rehabilitation and the Society for Academic Emergency Medicine. Circulation 2007; 116: e148-304.

8. Bertrand ME, Simoons ML, Fox KA, Wallentin LC, Hamm CW, McFadden E, et al. Management of acute coronary syndromes in patients presenting without persistent STsegment elevation. The Task Force on the Management of Acute Coronary Syndromes of the European Society of Cardiology. Eur Heart J 2002; 23: 1809-40.

9. Van de Werf F, Bax J, Betriu A, Blomstrom-Lundqvist C, Crea F, Falk V, et al. Management of acute myocardial infarction in patients presenting with persistent STsegment elevation: the Task Force on the Management of ST-Segment Elevation Acute Myocardial Infarction of the European Society of Cardiology. Eur Heart J 2008; 29: 2909-45.

10. López Bescós L, Arós Borau F, Lidón Corbi RM, Cequier Fillat A, Bueno H, Alonso JJ, et al. Spanish Society of Cardiology. [2002 Update of the Guidelines of the Spanish Society of Cardiology for Unstable Angina/Without ST-Segment Elevation Myocardial Infarction]. Rev Esp Cardiol 2002; 55: 631-42.

11. Ndrepepa G, Mehilli J, Schulz S, Iijima R, Keta D, Byrne RA, et al. Patterns of presentation and outcomes of patients with acute coronary syndromes. Cardiology 2009; 113: 198-206.

12. Corbalán R, Nazzal C, Eggers G, Bartolucci, Prieto JC, Stockins B, et al. Resultados del primer registro chileno de angina inestable: características clínicas, perfil de riesgo y tratamiento. Rev Med Chile 2004; 132: 135-43.

13. Hasdai D, Behar S, Wallentin L, Danchin N, Gitt AK, Boersma E, et al. A prospective survey of the characteristics, and outcomes of patients with acute coronary syndromes in Europe and the Mediterranean basin. The Euro Heart Survey of Acute Coronary Syndromes (Euro
Heart Survey ACS). Eur Heart J 2002; 23: 1190-201.

14. Ray KK, Cannon CP, McCabe CH, Cairns R, Tonkin AM, Sacks FM, et al. PROVE IT-TIMI 22 Investigators. Early and late benefits of high-dose atorvastatin in patients with acute coronary syndromes: results from the PROVE IT-TIMI 22 trial. J Am Coll Cardiol 2005; 46: 1405-10.

15. Braunwald E, Antman EM, Beasley JW, Califf RM, Cheitlin MD, Hochman JS, et al. ACC/AHA 2002 Guideline Update for the Management of Patients With Unstable Angina and Non-ST-Segment Elevation Myocardial Infarction-Summary Article. A Report of the American College of Cardiology / American Heart Association Task Force on Practice Guidelines (Committee on the Management of Patients With Unstable Angina). J Am Coll Cardiol 2002; 40: 1366-74.

16. Alexander D, Ou FS, Roe MT, Pollack CV Jr, Ohman EM, Cannon CP, et al. Use of and inhospital outcomes after early clopidogrel therapy in patient not undergoing an early invasive strategy for treatment of non-ST segment elevation myocardial infarction: Results from Can Rapid risk stratification of Unstable angina patients Suppress ADverse outcomes with Early implementation of the American College of Cardiology/American Heart Association guidelines (CRUSADE) Am Heart J 2008; 156: 606-12.

17. Rao RV, Goodman SG, Yan RT, Spencer FA, Fox KA, DeYoung JP, et al. Canadian Global Registry of Acute Coronary Events (GRACE/GRACE(2)) Investigators. Temporal trends and patterns of early clopidogrel use across the spectrum of acute coronary syndromes. Am Heart J 2009; 157: 642-50.

18. Fox KA, Dabbous OH, Goldberg RJ, Pieper KS, Eagle KA, Van de Werf F, et al. Prediction of risk of death and myocardial infarction in the six months after presentation with acute coronary syndrome: prospective multinational observational study (GRACE). BMJ 2006; 333 (7578): 1091.

19. Weintraub WD. Pharmacoeconomic concepts in antiplatelet therapy: understanding cost-effectiveness analyses using clopidogrel as an example. J Cardiovasc Pharmacol Ther 2008; 13: 107-19.

20. Prieto JC, Chávez E, Corbalán R, Yovanovich J, Cumsille F, Nazzal C. Acute myocardial infarction in Chile: differences between men and women in its evolution and prognosis. Multicenter Study Group on Infarction (GEMI). Rev Med Chile 1996; 124: 785-92.

21. Zia MI, Goodman SG, Peterson ED, Mulgund J, Chen AY, Langer A, et al. Paradoxical use of invasive cardiac procedures for patients with non-ST segment elevation myocardial infarction: an international perspective from the CRUSADE Initiative and the Canadian ACS Registries I and II. Can J Cardiol 2007; 23: 1073-9. 